\title{
ANALYSIS OF A CUSTOMER DEMAND DRIVEN SEMICONDUCTOR SUPPLY CHAIN IN A DISTRIBUTED SIMULATION TEST BED
}

\author{
Chin Soon Chong \\ Peter Lendermann \\ Boon Ping Gan \\ Production and Logistics Planning Group \\ Singapore Institute of Manufacturing Technology \\ 71 Nanyang Drive \\ Singapore 638075, SINGAPORE
}

\author{
Brett Marc Duarte \\ John W. Fowler \\ Thomas E. Callarman \\ Institute for Manufacturing Enterprise Systems \\ Department of Industrial Engineering \\ PO Box 875906 \\ Arizona State University \\ Tempe, AZ 85287-5906, U.S.A.
}

\begin{abstract}
Effective supply chain management (SCM) enables organizations to be more competitive in the current world of global manufacturing by reducing costs and improving the quality of customer service. Simulation can assist in moving towards these goals by evaluating the feasibility of alternative policies for managing a supply chain. However, simulation of multiple factories within the supply chain, with a high level of granularity in particular, can be very complex and computationally intensive. In this paper, we describe how a distributed simulation test bed enabling very detailed supply chain simulation can be used to study a customer-demand driven semiconductor supply chain.
\end{abstract}

\section{INTRODUCTION}

In today's increasingly globalized markets, manufacturers are permanently forced to look for ways to sharpen their competitive edge. These efforts can no longer be limited to the four walls of a factory but need to be extended to the entire supply chain (Jain et al. 2002, Venkateswaran et al. 2002). Manufacturers need to ensure that their operations integrate well into the supply chain so that products can reach the end-customers efficiently and cost-effectively. The need to have a competitive supply chain is particularly critical in the semiconductor industry, due to the large capital investment and the high value of end products (Jain et al. 1999) which have a short market life-span because of rapid changes in technology (Turner et al. 2000).

This calls for supply chain management (SCM), which involves planning and managing the flow of material and information through multiple stages of manufacturing, transportation and distribution. SCM involves multiple echelons, processes and organizational functions that coordinate and integrate together (Angerhofer and Angelides 2000). It includes inventory replenishment as well as plan- ning of operations at each manufacturing stage. It also includes planning of shipments for products from one stage to the next. SCM can help manufacturers to maintain high utilization of equipment with minimal inventory while improving customer service performance and profitability.

Since supply chains are often large systems consisting of many entities interacting in complex ways (Barnett and Miller 2000), managing a supply chain and finding the right strategy across the supply chain is a challenging task (Truong and Azadivar 2003). Hence, analysis tools and methodologies that can help in managing a supply chain efficiently are increasingly important (Chwif et al. 2002). Two basic categories of approaches can be identified for managing supply chains: solution evaluation and solution generation (Harrel and Tumay 1994). The first category deals with evaluation of the possible configurations of a supply chain design whereas the second generates an optimal configuration for a given set of objectives. This paper focuses primarily on solution evaluation.

Among various tools and methodologies, discrete event simulation has been proven valuable as a practical tool for representing complex interdependencies, evaluating alternative designs and policies, and analyzing performance tradeoffs for supply chain systems (Hennessee 1998, Chwif et al. 2002, Jain et al. 2002, Venkateswaran et al. 2002, Enns and Suwanruji 2003, Gan et al. 2000). Simulation allows both material and information flow to be modeled through each of the stages in the supply chain, together with complex decision logic for planning, scheduling and control. In addition, simulation can handle stochastic demand patterns and environments, multiple criteria and objectives, etc.

The popularity of using simulation in managing supply chains is also reflected in the literature. For example, IBM developed a supply chain simulator, which comprises a mix of simulation and optimization functions, to model and analyze its own supply chain issues (Bagchi et al. 1998). IBM also used its own simulation-based supply chain analyzer to 
visualize and quantify the effects of making changes on a hypothetical supply chain, and the impact of the changes to the system performance (Archibald et al. 1999). i2 described a solution methodology for supply chains based on an integrated simulation and optimization approach (Padmos et al. 1999). Jain et al. (2001) described a simulation study on the supply chain for a large logistics operation. The results indicate that improvement in forecast accuracy can provide larger savings than process automation changes. The paper also pointed out the usefulness of simulation to evaluate the performance of supply chains.

The need for executing supply chain simulations based on a full-detail model has also been pointed out: Jain et al. (1999) compared two models of different level of detail for semiconductor manufacturing supply chains. In their paper, it is shown that simulations incorporating detailed models are required to determine the correct inventory levels for maintaining desired customer responsiveness. Abstracted models can give inaccurate results that may subsequently lead to erroneous decisions. Venkateswaran et al. (2002) drew similar conclusions in their paper. They used multiple models with varying degrees of detail representing the same supply chain. It is shown that the system dynamics vary with increasing detail in the model. It is concluded that a strategy found to be the most effective on an abstract model is not always the best for a detailed model. In another paper (Jain et al. 1999), it is stressed that highfidelity simulation is required for optimization of planning and execution of supply chains.

Simulation enables evaluation and analysis of complex systems such as those found in semiconductor supply chains, but as the model increases in size and realism, a more powerful simulation capability that is beyond the sequential execution approach is required. The High Level Architecture (HLA), which is a framework developed by the Defense Modeling and Simulation Office (DMSO), provides the necessary infrastructure for large-scale distributed simulation. In HLA, a federate can be viewed as a component simulation model that is taking part in a large simulation (Turner et al. 2000). A federation consists of a set of federates. In the case of supply chain simulation, federates can be factories in the supply chain and the federation is then the supply chain itself. In our work we have adopted this infrastructure in a distributed simulation test bed to improve on the simulation execution speed, to support reusability of existing simulation models and interoperability between different simulation packages. Applicability of distributed simulation for decision-making in semiconductor manufacturing has been demonstrated by Lendermann et al. (2003).

In this paper, we describe the simulation test bed and report on the results of analysis for a semiconductor supply chain using the test bed. The next section explains the supply chain simulation model used for the study. Section 3 describes the simulation test bed. Section 4 discusses the experiments that we performed using the test bed and their results, followed by the conclusions in Section 5 .

\section{SEMICONDUCTOR SUPPLY CHAIN MODEL}

The simulation model used in our study comprises the basic elements of a supply chain (Jain et al. 1999). These elements include manufacturing, transportation, business processes and customer orders as depicted in Figure 1. Two successive stages of material transformation, from a wafer fabrication facility to an assembly and test facility, are modeled. The wafer fabrication facility is the supplier to the assembly and test facility, which subsequently supplies Integrated Circuits (ICs) to end-customers. The transportation of wafers between the two manufacturing stages and the transportation of ICs to customers are modeled as well. Forecasting, production and inventory planning that are related to business processes are incorporated in the model. Customer orders are generated with the actual rate allowed to be different from the forecasted rate so as to simulate real life situations.

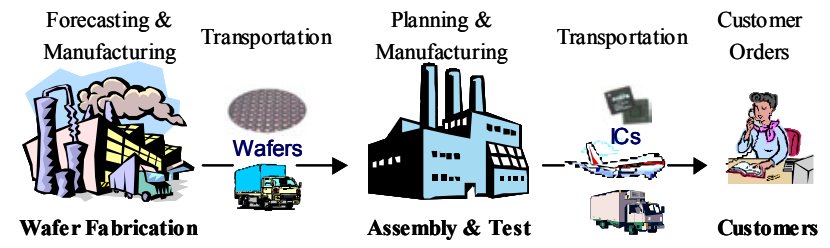

Figure 1: Scope of the Semiconductor Supply Chain

The wafer fabrication plant data is based on factory data from Sematech dataset 1, which is available through the Internet (MASMLab 2004). The data for the assembly and test facility is based on past projects with the industry. The data, particularly volume release and factory capacities, has been adapted to ensure that the production quantities and the utilization of facilities are consistent with what is typically found in the industry. In our supply chain model, the production of wafers in the wafer fabrication facility is based on a make-to-stock strategy driven by forecast, whereas the production of ICs in the assembly and test facility is based on a make-to-order strategy. The volume release rate of wafers is varied periodically (i.e. weekly, bi-weekly, monthly, etc.) based primarily on the forecasted demand and the availability of wafers in the supply chain. The lot release in assembly and test facility is driven by actual customer order arrivals. For further details, refer to the paper by Jain et al. (1999).

The major components in the supply chain model and the interactions between them are summarized in Figure 2. In the supply chain, the federates interact with each other through information and material flow. The information flow is shown using dashed lines while the material flow is shown using solid lines. There are four federates in the supply chain:

1. Demand Generator $(\mathrm{D} / \mathrm{G})$ - Customer order generator

2. Lot Allocation Engine (LAE) - Order planning 
3. Wafer Fabrication (W/F) - Wafer fabrication facility

4. Assembly \& Test (A\&T) - Assembly and test facility

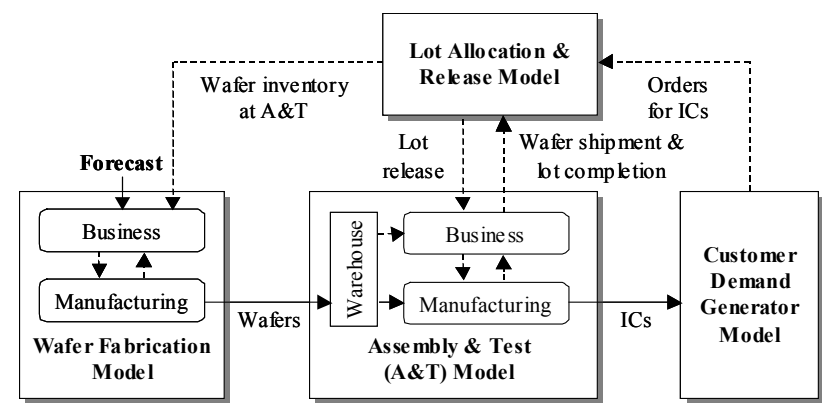

Figure 2: Interactions between Components of Semiconductor Supply Chain

Both the W/F and the A\&T factory have a business process and a manufacturing process. The manufacturing process simulates the transformation of raw materials into products while the business process simulates production planning and inventory management. The model currently simulates two types of wafer products, and each of these products is used to manufacture two different types of ICs (i.e. a total of four types of ICs) in the A\&T facility (see Table 1).

Table 1: Scenario Considered in the Supply Chain Model

\begin{tabular}{|c|c|c|c|c|}
\hline $\begin{array}{c}\text { A\&T } \\
\text { Product } \\
\text { (IC) }\end{array}$ & $\begin{array}{l}\text { A\&T Order } \\
\text { Size }\end{array}$ & \begin{tabular}{|c|} 
Wafer Lot \\
Size
\end{tabular} & $\begin{array}{l}\text { Die Per } \\
\text { Wafer }\end{array}$ & \begin{tabular}{|c|} 
Wafer \\
Product
\end{tabular} \\
\hline $\mathrm{P} 1$ & $21660 \pm \mathrm{n} \%$ & $\leq 24$ & 200 & $\mathrm{~W} 2$ \\
\hline $\mathrm{P} 2$ & $20660 \pm \mathrm{n} \%$ & $\leq 24$ & 210 & $\mathrm{~W} 2$ \\
\hline P3 & $20830 \pm \mathrm{n} \%$ & $\leq 24$ & 200 & W1 \\
\hline P4 & $20875 \pm \mathrm{n} \%$ & $\leq 24$ & 200 & W1 \\
\hline
\end{tabular}

The $\mathrm{D} / \mathrm{G}$ generates customer orders for each type of IC on a daily basis. These orders are fed to the LAE, which then assigns and releases orders to A\&T based on the availability of factory capacity and wafer inventory in A\&T. Fabricated wafers are shipped to the A\&T warehouse on a daily basis with a shipment delay of a day. The wafers in the warehouse are used to produce ICs in the A\&T facility, and the completed and packaged ICs are transported to customers with another day's delay. A more detailed description of each federate is provided in the following subsections.

\subsection{Demand Generator (D/G)}

The D/G generates customer orders daily based on a predefined demand profile for each type of ICs. The volume for the orders of ICs can be varied each week by altering the demand profile. Each order is randomly assigned to a customer and due dates are randomly assigned to each customer order, based on a uniform distribution. The order information is sent to the LAE daily with a one-day delay (i.e. to simulate daily planning in the LAE).

\subsection{Lot Allocation Engine (LAE)}

The LAE receives customer orders from $\mathrm{D} / \mathrm{G}$, plans the orders in standard lot sizes and subsequently sends the lot release information to A\&T for the actual release of the product into the factory. In the order planning process, two major issues are involved. The first issue is to allocate A\&T factory capacity to the orders (i.e. capacity planning), and the second issue is to assign wafer lots available in the warehouse of A\&T to the orders. The order planning procedure is adapted from the lot-to-order matching policies described in the papers by Knutson et al. (1999) and Fowler et al. (2000). We have extended the matching policies to deal with multiple products for our supply chain model, instead of single product policies as depicted in the papers. The planning procedure is as follows:

1. Order Prioritization: The heuristic dynamically ranks and prioritizes orders accumulated on a given day based on a function of the order size, its lateness, the customer priority and the product priority.

2. Ranking of wafer lots in decreasing lot size: Lots for each wafer product in the warehouse of $A \& T$ are ranked in decreasing order based on the lot size.

3. Allocation of factory capacity and assignment of wafer lots to orders: For each customer order in the ranked list, the factory capacity and the wafer lots are first checked for availability to satisfy the order requirement. If both the capacity and the wafer lots are sufficient for the requirement, the order will then be allocated and assigned with the capacity and the wafer lots respectively before it is released into the factory. The actual size of a released order will always be greater than or equal to that of the actual customer order size due to the lot-toorder matching policies. The additional ICs produced in the order are termed Die-To-Warehouse (DTW) inventory

4. Release of A\&T orders in standard lot sizes: Orders are released into the shop floor at regular intervals based on standard lot sizes. Each A\&T product has its standard size as provided in Table 1. The lots that complete all the A\&T process steps are accumulated to reform the original orders. The completed orders are then sent to customers.

\subsection{Wafer Fabrication (W/F)}

As mentioned earlier, the $\mathrm{W} / \mathrm{F}$ is represented by a Sematech data set and produces two wafer products, which 
go through 210 and 245 process steps respectively. There are 32 operator groups in the dataset. The primary dispatching rules for machines are FIFO and Setup Avoidance (only in medium and high current implantation machines). Wafers processed in the $\mathrm{W} / \mathrm{F}$ are then sent to an inventory hold in the A\&T. A transportation delay of 1 day is required for this shipment. W/F releases wafer lots into production based on the product's work-in-progress level in the factory and in transit, inventory level of the wafer product in the warehouse of $A \& T$, the desired safety stock level and the forecasted demand of the product. Adjustments to the release quantities in the $\mathrm{W} / \mathrm{F}$ are based on the inventory updates, which occur at fixed time intervals and can be weekly, bi-weekly, monthly, etc. Wafer releases can be summarized by the following formula:

Wafer release quantity $=$ Forecast demand - Excess inventory

where

Excess Inventory $=\sum$ (Work-in-progress in W/F, WIP in transit, Wafers remaining) - $\sum$ (Safety stock level, and Cumulative demand from current to target period)

Also,

Wafer remaining $=$ Wafer inventory in the A\&T - Wafers required for all 'unplanned' orders

The cumulative demand is computed based on the sum of the forecast demands from current period to the target period. The number of periods in the summation is dependent on the average cycle time of the product considered. The stated wafer release formula is slightly different from the version found in the paper by Jain et al. (1999). In our formula, we have introduced safety stock level and considered wafer inventory shortage. The yield loss during wafer production process has also been incorporated into the forecast.

\subsection{Assembly and Test (A\&T)}

The A\&T dataset has four products and each product has approximately 25 process steps. A\&T receives lot release commands from LA and releases lots based on the predetermined release times and sizes determined by LA. The major dispatching rule for machines in A\&T is FIFO.

\section{SIMULATION TEST BED}

The simulation test bed used for this study is an HLAbased discrete event simulation system that originated from a semiconductor supply chain simulator developed in $\mathrm{C}++$ under a joint project between Singapore Institute of Manu- facturing Technology and the School of Computer Engineering at Nanyang Technological University, Singapore (Jain et al. 1999). The test bed is implemented using the Run Time Infrastructure (RTI), which is an implementation of the HLA Interface Specification (Turner et al. 2000). In our model, each sub-model of the supply chain maps to a federate in the supply chain federation. Each federate in the federation is executed on separate process in workstations and can be geographically distributed.

Since the supply chain model is developed modularly using the HLA, it is straightforward to replace any federate with a new one as long as the interface format standard is adhered to. In our test bed, we have made the demand generator and production planning separate federates so as to realistically simulate what is commonly happening in industry. In semiconductor manufacturing supply chains, the locations of customers, planning centers and manufacturing facilities can be geographically dispersed. Another reason for making them federates is to later replace them by commercial tools in a 'plug and play' manner.

Parameter files are used to define the overall scope of the distributed simulation. In addition, each federate has its own parameter files for defining the specific configuration of the federate in the supply chain. The parameter files for the federate can be used to define dispatch and setup rules for each machine set, priority of products, weekly demand profile, safety stock level, and so on. The factory federates also have the Sematech format files, which are used to specify process routes, resources such as machines and operators, resource unavailable times such as down times and break-times, and volume release of products.

As the simulation of the supply chain starts from an empty factory condition, a special warm-up policy is needed to ensure that the simulation can reach steady state conditions in a much shorter time. During the warm-up period, as long as the A\&T facility has not received any lots of a particular wafer product, we allow the corresponding A\&T product to be released immediately. After the first lot of the wafer product arrives at the warehouse, we continue to assume having sufficient stock until one month after the first arrival of the product. This policy also helps to avoid a large number of unfilled customer orders queuing in the LAE.

\section{EXPERIMENTATION}

In our study, we examine the on-time-delivery (OTD) performance of the A\&T facility. OTD is defined as the percentage of completed lots that reach customers on or before the due dates. Due dates are randomly assigned to orders with a specific percentage of variation based on a mean value of 8 days. All experiments are performed with multiple replications (8 runs) using different random streams to determine the performance in each case with a certain level of confidence. The simulation runs are conducted for the model for periods of 6 years with a 3 year warm up. 


\subsection{Frequency of Inventory Update}

Figure 3 shows the OTD performance for different inventory information feedback and update intervals, ranging from 1 week to 12 weeks. No general trend on the performance can be identified with increasing feedback and update interval from the graph. However, an investigation into the standard deviations of WIP and inventory of the wafer products indicates that there is an increasingly larger fluctuation of WIP and inventory with increasing update interval. This can be explained by considering the fact that lot cycle times in W/F is about 8 times that of A\&T. Given Little's law, this also implies that WIP in W/F is approximately 8 times that of A\&T's. This WIP can thus act as a large buffer for the deviation between actual wafer lot release in $\mathrm{W} / \mathrm{F}$ and the required wafers in A\&T for the long update interval cases. Furthermore, at the beginning of simulation, the low initial inventory level in A\&T causes more wafer lots to be released continuously, and this results in large WIP being built up for the long update interval cases. This large WIP will then be consumed during the next inventory update when total WIP is found to be excessive, causing less wafer lots to be released. This "vicious" cycle of large fluctuation of WIP and inventory repeats itself.

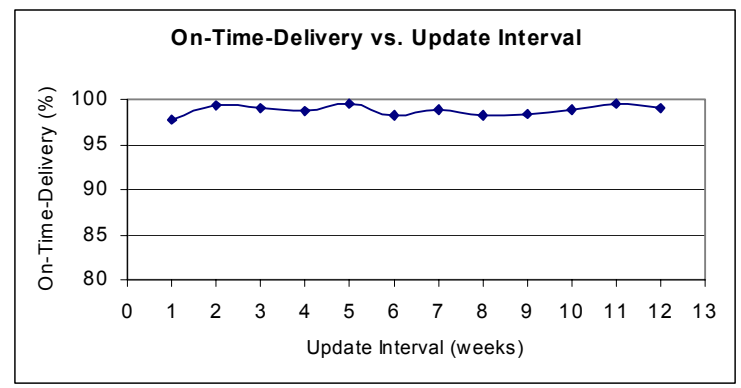

Figure 3: Time-Delivery vs. Update Interval

\subsection{Demand Variability}

Figure 4 shows the effect of increasing demand variation on the performance. From the results, it is obvious that increasing variation in demand deteriorates OTD. This can be explained by considering the impact of the order size variation on the inventory in A\&T and subsequently WIP in $\mathrm{W} / \mathrm{F}$ based on the wafer release formula.

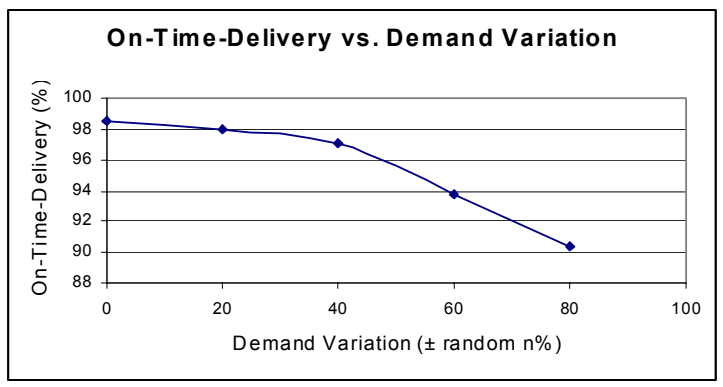

Figure 4: On-Time-Delivery vs. Demand Variation

\subsection{Due-Date Variability}

Figure 5 shows the effects of random variation of due dates on the performance. With the inventory threshold level fixed at a certain level, OTD decreases with increasing due date variations around a pre-defined due date of 8 days (from $\pm 0 \%$ to $\pm 90 \%$ ). Increasing variation of due date at the early stage (i.e. from $\pm 0 \%$ to $\pm 30 \%$ ) has negligible impact on the OTD performance. This implies that the supply chain model can accommodate due date variation up to $30 \%$. Further variation of due dates requires higher inventory threshold levels to achieve good OTD performance.

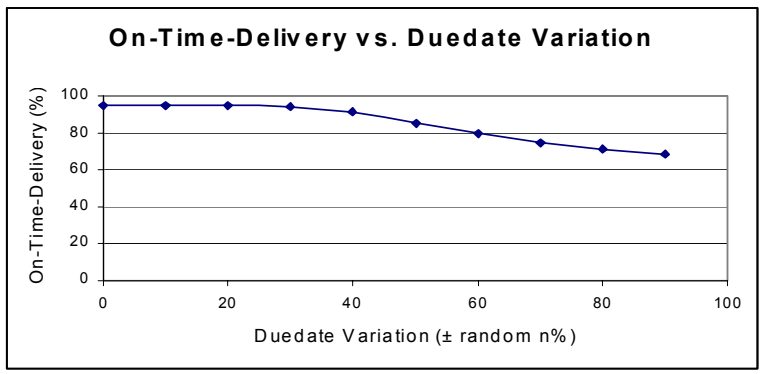

Figure 5: On-Time-Delivery vs. Due Date Variation

\subsection{Dispatching Priority for the Last $n$ Steps of Wafer Lots in W/F}

A new dispatching rule has been introduced to rank the wafer lots for the last ' $n$ ' steps of product routings in W/F based on the demand and availability of that particular product in inventory. An Inventory threshold level $I_{T}$ can be arbitrarily set for each wafer product. For those wafer products with inventory levels greater than the thresholds, First-In-First-Out or Setup Avoidance rule is used as in the original model. However, if the inventory level falls below the threshold level, then that particular wafer product is given a higher priority in the backend (last ' $n$ ' steps) of the $\mathrm{W} / \mathrm{F}$. Further, lots that are closer to the end of their process flow are assigned higher priority than those at the beginning of the process flow if the lots share the same machine groups for processing. This ensures that the required wafer products reach AT as soon as possible.

A simulation experiment was performed by applying the dispatching rule at different stages in the W/F and setting the inventory level of $\mathrm{W} 1$ at a high level and that of W2 at a sufficient level. A demand spike (approximately 2 times the original demand) is introduced for a period of 9 weeks into one of the A\&T products, which requires W2. Inventory threshold levels are set to 100 wafers for both wafer products (100 wafers were chosen based on earlier experiments with different inventory levels). The purpose of introducing the demand spike is to study the impact of a temporary demand change on the supply chain and to investigate the effectiveness of the new dispatching rule on the disruption. This scenario simulates a temporary demand change in the industry. 
The simulation result is shown in Figure 6. The dots on the graphs are the OTD performance for steady demand cases. When a demand spike is introduced, the system performance deteriorates (i.e. last 0 steps). However, when the new dispatching rule is applied in $\mathrm{W} / \mathrm{F}$ (i.e. from last 20 steps onwards), OTD improves. The performance eventually reaches an optimum (i.e. OTD 1) as the rule is used in more and more processing steps of lots. This result can be explained by considering the impact of the dispatching rule on the two wafer products. The dispatching rule favors W2 over W1 initially due to excess inventory of $\mathrm{W} 1$, but when too many lots use the dispatching rule, the rule eventually alternates between $\mathrm{W} 1$ and $\mathrm{W} 2$. This occurs when inventory level of W1 drops to below a certain threshold and that of W2 reaches a certain threshold. To further verify our reasoning, we switched the dispatching rule trigger off for W1. The plot shows that OTD performance worsens after reaching an optimum (i.e. OTD 2).

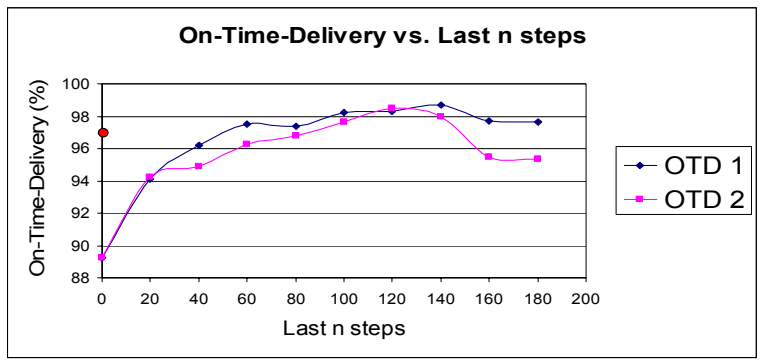

Figure 6: On-Time-Delivery vs. Last n Steps

\section{CONCLUSIONS}

This paper described the use of a detailed distributed simulation model for the analysis of a semiconductor supply chain. The study has provided quantified estimates of the performance. From our study, the following conclusions can be made:

- The impact of varying inventory update interval on OTD and tardiness performance is insignificant, although the effect on WIP and inventory (i.e. variance) is substantial.

- Increasing variations of demand and due date cause OTD performance to deteriorate. These variations play an important role in the performance of the supply chain (Chwif et al. 2002).

- Assigning higher priority for wafer products that are 'short' in the warehouse of A\&T can improve OTD performance. However, this method tends to cause other wafer products to be compromised.

The paper also demonstrates the usefulness of distributed simulation to evaluate the performance of supply chains. The use of an integrated model of information and material flows, as well as business and manufacturing processes en- ables capturing very detailed aspects of operations and interaction of individual entities in the supply chain. However, the results in this study are based on a hypothetical supply chain and therefore the results cannot be validated against the real system. To obtain the true characteristics of an actual system, it is recommended that supply chain simulations be validated against real supply chain performance.

\section{ACKNOWLEDGMENTS}

This research is funded by The Institute for Manufacturing Enterprise Systems (IMES), Modeling and Analysis for Semiconductor Manufacturing Laboratory (MASMLAB), both of Arizona State University (ASU), Tempe, USA, and SIMTech, Singapore. The research is supported under a collaborative project, entitled Analysis of Coordination Mechanisms and Relationship in Supply Chain Using Distributed Simulation, between ASU and SIMTech.

\section{REFERENCES}

Angerhofer, B.J., M.C. Angelides. 2000. System dynamics modeling in supply chain management: Research review. In Proceedings of the 2000 Winter Simulation Conference, ed. J.A. Joines, R.R. Barton, K. Kang and P.A. Fishwick, 342-351. Piscataway, NJ: IEEE.

Archibald, G., N. Karabakal, and P. Karlsson. 1999. Supply chain vs. supply chain: Using simulation to compete beyond the four walls. In Proceedings of the 1999 Winter Simulation Conference, ed. Farrington, H.B. Nembhard, D.T. Sturrock, and G.W. Evans, 888-896. Piscataway, NJ: IEEE.

Bagchi, S., S.J. Buckley, M. Ettl, and G.Y. Lin. 1998. Experience using the IBM supply chain simulator. In Proceedings of the 1998 Winter Simulation Conference, ed. D.J. Medeiros, E.F. Watson, J.S. Carson and M.S. Manivannan, 1387-1394. Piscataway, NJ: IEEE.

Barnett, M.W., and C.J. Miller. 2000. Analysis of virtual enterprise using distributed supply chain modeling and simulation: An application of e-SCOR. In Proceedings of the 2000 Winter Simulation Conference, ed. J.A. Joines, R.R. Barton, K. Kang and P.A. Fishwick, 352355. Piscataway, NJ: IEEE.

Chwif, L., M.R.P. Barretto, E. Saliby. 2002. Supply chain analysis: Spreadsheet or simulation? In Proceedings of the 2002 Winter Simulation Conference, ed. E. Yücesan, C.-H. Chen, J.L. Snowdon, and J.M. Charnes, 5966. Piscataway, NJ: IEEE.

Enns, S.T., and P. Suwanruji. 2003. A simulation test bed for production and supply chain modeling. In Proceedings of the 2003 Winter Simulation Conference, ed. S. Chick, P.J. Sánchez, D. Ferrin, and D.J. Morrice, 1174-1182. Piscataway, NJ: IEEE.

Fowler, J., K. Knutson, and M. Carlyle. 2000. Comparison and evaluation of lot-to-order matching policies for a 
semiconductor assembly and test facility. International Journal of Production Research. 38(8): 1841-1853.

Gan, B.P., L. Liu, S. Jain, S.J. Turner, W. Cai, and W.J. Hsu. 2000. Distributed supply chain simulation across enterprise boundaries. In Proceedings of the 2000 Winter Simulation Conference, ed. J.A. Joines, R.R. Barton, K. Kang and P.A. Fishwick, 1245-1251. Piscataway, NJ: IEEE.

Harrel, C., and K. Tumay. 1994. Simulation made easy. Engineering \& Management press.

Hennessee, M. 1998. Challenges facing global supplychains in the $21^{\text {st }}$ century. In Proceedings of the 1998 Winter Simulation Conference, ed. D.J. Medeiros, E.F. Watson, J.S. Carson and M.S. Manivannan, 3-4. Piscataway, NJ: IEEE.

Jain, S., C.C. Lim, B.P. Gan, and Y.H. Low. 1999. Criticality of detailed modeling in semiconductor supply chain simulation. In Proceedings of the 1999 Winter Simulation Conference, ed. P.A. Farrington, H.B. Nembhard, D.T. Sturrock, and G.W. Evans, 888-896. Piscataway, NJ: IEEE.

Jain, S., E.C. Ervin, A.P. Lathrop, R.W. Workman, L.M. Collins. 2001. Analyzing the supply chain for a large logistics operation using simulation. In Proceedings of the 2001 Winter Simulation Conference, ed. B.A. Peters, J.S. Smith, D.J. Medeiros, and M.W. Rohrer, 1123-1128. Piscataway, NJ: IEEE.

Jain, S., N.F. Choong, and W. Lee. 2002. Modeling computer assembly operations for supply chain integration. In Proceedings of the 2002 Winter Simulation Conference, ed. E. Yücesan, C.-H. Chen, J.L. Snowdon, and J.M. Charnes, 1165-1173. Piscataway, NJ: IEEE.

Knutson, K., K. Kempf., J.W. Fowler, and M. Carlyle. 1999. Lot-to-order matching for a semiconductor assembly \& test facility. IIE Transactions on Scheduling and Logistics, 31(11): 1103-1111.

Lendermann, P., N. Julka, B.P. Gan, D. Chen, L.F. McGinnis, and J.P. McGinnis. 2003. Distributed Supply Chain Simulation as a Decision-Support Tool for the Semiconductor Industry, Simulation, 79(3): 126-138.

MASMLab. 2004. Test-Bed [Online], maintained by Modeling and Analysis of Semiconductor Manufacturing Laboratory, Industrial and Management Systems Engineering department, Arizona State University, USA. Available: http://www.eas.asu.edu/ masmlab/ftp.htm [2004, Jan 31]

Padmos, J., B. Hubbard, T. Duczmal, and S. Saidi. 1999. How i2 integrates simulation in supply chain optimization. In Proceedings of the 1999 Winter Simulation Conference, ed. Farrington, H.B. Nembhard, D.T. Sturrock, and G.W. Evans, 888-896. Piscataway, NJ: IEEE.

Truong, T.H., and F. Azadivar. 2003. Simulation based optimization for supply chain configuration design. In Proceedings of the 2003 Winter Simulation Conference, ed. S. Chick, P.J. Sánchez, D. Ferrin, and D.J. Morrice, 1174-1182. Piscataway, NJ: IEEE.
Turner, S.J., W. Cai, and B.P. Gan. 2000. Adapting a supply chain simulation for HLA. In Proceedings of IEEE $4^{\text {th }}$ International Workshop on Distributed Simulation and Real Time Applications, 71-78, San Francisco, USA.

Venkateswaran, J., Y.-J. Son, and B. Kulvatunyou. 2002. Investigation of influence of modeling fidelities on supply chain dynamics. In Proceedings of the 2002 Winter Simulation Conference, ed. E. Yücesan, C.-H. Chen, J.L. Snowdon, and J.M. Charnes, 1183-1191. Piscataway, NJ: IEEE.

\section{AUTHOR BIOGRAPHIES}

CHIN SOON CHONG obtained his degree in Electrical and Electronics Engineering from the City University of London, UK. He joined Singapore Institute of Manufacturing Technology (SIMTech), a research institute in Singapore, twelve years ago and is currently in the Production and Logistics Planning Group. He obtained his Master of Engineering in Computer Integrated Manufacturing from Nanyang Technological University, Singapore. He is certified by the American Production and Inventory Control Society as a Certified Production and Inventory Manager (CPIM). During twelve years in SIMTech, he has been involved in simulation, scheduling and optimization related projects in logistic and manufacturing IT domains. The industrial projects include cargo container operation and yard capacity planning simulation, dairy-printing process simulation, manufacturing cycle-time modeling, scheduling and optimization for various MNCs. His current research interests include intelligent and integrated simulation, planning, scheduling, optimization in the area of manufacturing, logistics and supply chain. His email address is $<$ CsChong@ SIMTech.a-star.edu.sg>

PETER LENDERMANN is a Senior Scientist in the Production and Logistics Planning Group at Singapore Institute of Manufacturing Technology (SIMTech). Previously he was a Managing Consultant with agiConsult in Germany where his focus was on the areas of supply chain management and production planning. He also worked as a Research Associate at the European Laboratory for Particle Physics CERN in Geneva (Switzerland) and Nagoya University (Japan). He obtained a Diploma in Physics from the University of Munich (Germany), a Doctorate in Applied Physics from Humboldt-University in Berlin (Germany) and a Master in International Economics and Management from Bocconi-University in Milan (Italy). His research interests include parallel and distributed simulation and advanced methods for supply chain planning and production scheduling. His email address is peterl@SIMTech. a-star.edu.sg>

BOON PING GAN is a Senior Research Engineer with the Production and Logistics Planning Group at Singapore 
Institute of Manufacturing Technology (formerly known as Gintic Institute of Manufacturing Technology). He is currently leading a research project that attempts to apply distributed simulation technology for supply chain simulation. He received a Bachelor of Applied Science in Computer Engineering and Master of Applied Science from Nanyang Technological University of Singapore in 1995 and 1998 respectively. His research interests are parallel and distributed simulation, parallel programs scheduling, and application of genetic algorithms. His email address is <bpgan@ sIMTech.a-star.edu.sg>

BRETT MARC DUARTE is a PhD student in Industrial Engineering at Arizona State University. He has masters degree in Industrial Engineering with a specialization in the manufacture of semiconductors, and his interests lie in simulation and modeling, with an emphasis on supply chain management and integration. His email address is $<$ Brett.Duarte@asu.edu $>$

JOHN W. FOWLER is a Professor in the Industrial Engineering Department at Arizona State University. Prior to his current position, he was a Senior Member of Technical Staff in the Modeling, CAD, and Statistical Methods Division of SEMATECH. He received his Ph.D. in Industrial Engineering from Texas A\&M University and spent the last 1.5 years of his doctoral studies as an intern at Advanced Micro Devices. His research interests include modeling, analysis, and control of semiconductor manufacturing systems. Dr. Fowler is the co-director of the Modeling and Analysis of Semiconductor Manufacturing Laboratory at ASU. The lab has had research contracts with NSF, SRC, SEMATECH, Infineon Technologies, Intel, Motorola, ST Microelectronics, and Tefen Ltd. He is a member of ASEE, IIE, IEEE, INFORMS, POMS, and SCS. His email address is $<$ john.fowler@asu.edu>

THOMAS E. CALLARMAN is an Associate Professor of Supply Chain Management and has been on the faculty of Arizona State University since 1980. Dr. Callarman received his bachelor's degree in Business Administration from West Texas State University, his MBA from Arizona State University and his Ph.D. from Purdue University. Currently, he teaches Operations and Logistics Management in the ASU \& MBA High Technology program programs, and he previously served as the Associate Dean for Student Support in the Graduate College. He is currently the Director of the Institute for Manufacturing Enterprise Systems. Dr Callarman has published articles in Decision Sciences, the European Journal of Operational Research, the International Journal of Purchasing and Materials Management, and other research and practitioner journals. His current research interests are in the areas of process analysis and process improvement. He is president-elect of the Decision Science Institute. His email address is <tom. callarman@asu.edu> 